\title{
Impacto do DSM-5 no Diagnóstico de Transtornos de Aprendizagem em Crianças e Adolescentes com TDAH: Um Estudo de Prevalência
}

\author{
The Impact of DSM-5 on the Diagnosis of Learning Disorder \\ in ADHD Children and Adolescents: A Prevalence Study
}

\author{
Beatriz Vargas Dorneles ${ }^{*}, a$, Luciana Vellinho Corso ${ }^{a}$, Adriana Corrêa Costa ${ }^{a}$, \\ Nelba Maria Teixeira Pisacco ${ }^{b}$, Yasmini Lais Spindler Sperafico ${ }^{a} \&$ Luis Augusto Paim Rohde ${ }^{a}$ \\ ${ }^{a}$ Universidade Federal do Rio Grande do Sul, Porto Alegre, RS, Brasil \\ $\&{ }^{b}$ Universidade Estadual de Ponta Grossa, Ponta Grossa, PR, Brasil
}

\begin{abstract}
Resumo
Estudos de prevalência de Transtornos de Aprendizagem (TA) em crianças e adolescentes com TDAH apresentam resultados não conclusivos, apesar da frequente comorbidade entre as síndromes. Esse artigo descreve e analisa a prevalência de TA em uma amostra referida de 270 crianças e adolescentes com TDAH atendidas no Programa de Transtornos de Déficit de Atenção/Hiperatividade/Hospital de Clínicas de Porto Alegre - Universidade Federal do Rio Grande do Sul (ProDAH/HCPA-UFRGS), de acordo com os critérios diagnósticos do Manual Diagnóstico e Estatístico de Transtornos Mentais $I V$ - versão revisada (DSM-IV-TR). Verificou-se que 46,7\% dos estudantes apresentam, pelo menos, um TA, sendo o Transtorno da Expressão Escrita o mais frequente (32,6\%). Discutiu-se o potencial impacto das mudanças nos critérios diagnósticos para os TA, propostas pelo DSM-5 na prevalência desses transtornos. Embora seja possível afirmar que ocorrerão alterações nas taxas de prevalência de TA, o impacto do DSM-5 sobre a prevalência, especificidade e comorbidade do TA permanece incerto, já que há mudanças que ampliarão a abrangência dos critérios de inclusão e outras que a reduzirão. Palavras-chave: TDAH, transtornos de aprendizagem, comorbidade, diagnóstico, DSM-5.
\end{abstract}

\begin{abstract}
Prevalence studies on learning disabilities (LD) in children and adolescents with Attention Deficit and Hyperactivity Disorder (ADHD) show inconclusive results, despite the frequent comorbidity between these syndromes. This article describes and analyzes the prevalence of LD in a referred sample of 270 children and adolescents with ADHD treated at Disorders Program of Attention Deficit / Hyperactivity / Hospital de Clínicas de Porto Alegre - Federal University of Rio Grande do Sul (PRODAH/HCPA-UFRGS), according to the diagnostic criteria of the Diagnostic and Statistical Manual of Mental Disorders (DSM-IV-TR). The prevalence of LD among the sample was of $46.7 \%$. In that group, the most common LD was the Written Expression Disorder (32.6\%). The impact of the changes in the diagnostic criteria for LD proposed by the DSM-5 in the prevalence of these disorders is discussed. Although changes will occur in the rates of LD prevalence, the impact of the DSM-5 on the prevalence, specificity and comorbidity of LD remains uncertain, as there are some proposed changes that will broaden the scope of the criteria and others that will reduce it.

Keywords: ADHD, learning disorders, comorbidity, diagnosis, DSM-5.
\end{abstract}

O Transtorno de Déficit de Atenção Hiperatividade (TDAH) é um transtorno neurobiológico com etiologia multifatorial incluindo fatores genéticos e ambientais. Sua prevalência mundial é de cerca de $5,29 \%$ em crianças e adolescentes (Polanczyk, Lima, Horta, Biederman, \& Ro-

*Endereço para correspondência: Faculdade de Educação, Universidade Federal do Rio Grande do Sul, Programa de Pós-graduação em Educação, Av. Paulo Gama, s/n, $7^{\circ}$ andar, sala 700-08, Porto Alegre, RS, Brasil 90046-900.

E-mail: bvdornel@terra.com.br hde, 2007). Além disso, é um dos transtornos mentais da infância e da adolescência que determina grande procura por atendimento médico e psicopedagógico (Costa, 2009; Rohde, Dorneles, \& Costa, 2006). O transtorno é, em geral, evidenciado na infância, e frequentemente acompanha o indivíduo durante a vida adulta.

O desempenho escolar pode estar comprometido nas crianças com déficits de atenção significativos, associados ou não à hiperatividade, já que a atenção seletiva a informações relevantes é condição necessária para que ocorra aprendizagem, especialmente as escolares (DuPaul, 
Gormley, \& Laracy, 2012; Zentall, 2007). Estudos (Barkley, 2008; Rief, 2006) indicam que os estudantes com TDAH correm de duas a três vezes mais risco de fracassar na escola do que crianças sem TDAH e com inteligência equivalente. Na idade escolar, esses estudantes apresentam maior probabilidade de repetência, evasão escolar, baixo rendimento acadêmico e dificuldades emocionais e de relacionamento social (Carroll, Maughan, Goodman, \& Meltzer, 2005; Rohde et al., 1999).

Há evidências de que sintomas de desatenção são preditores de comprometimento em vários domínios da vida escolar (Lee, Lahey, Owens, \& Hinshaw, 2008; Wu \& Gau, 2013). Independentemente da persistência do transtorno, também há evidências de que o comprometimento do desempenho global na escola durante a infância mantém-se na adolescência (Wu \& Gau, 2013).

Alguns autores (Faraone, Biederman, Monuteaux, Doyle, \& Seidman, 2001; Mayes, Calhoun, \& Crowell, 2000) têm justificado esse pior desempenho pela alta prevalência de comorbidade entre os transtornos de aprendizagem (TA) e o TDAH. Vários modelos teóricos têm sido propostos para justificar tal comorbidade, sendo que três deles têm recebido maior atenção.

O primeiro sugere que os dois transtornos compartilham um ou mais déficits cognitivos, subjacentes a ambos, que justificam a presença dos dois, por exemplo, déficits na memória de trabalho e na velocidade de processamento da informação (DuPaul et al., 2012; Martinussen, Hayden, Hogg-Johnson, \& Tannock, 2005; Willcutt, Pennington, Olson, Chhabildas, \& Hulslander, 2005).

O segundo modelo propõe que a presença de um transtorno aumenta o risco para o outro, isto é, os três sintomas nucleares do TDAH, desatenção, agitação e impulsividade, têm um impacto forte na aprendizagem, pois levam os estudantes a trabalharem individualmente, de forma produtiva, por menos tempo que seus colegas sem TDAH. Esses alunos também apresentam dificuldade de engajamento em tarefas, constantemente prolongando o início de uma atividade, além de evitarem o treino repetitivo e não desenvolverem habilidades de forma tão automática quanto seus pares sem TDAH. Assim, os principais sintomas do TDAH afetam de tal forma a vida acadêmica das crianças que elas acabam por apresentar, como consequência, dificuldades de aprendizagem (DuPaul et al., 2012; DuPaul \& Stoner, 2007).

E o terceiro modelo sugere que a comorbidade representa transtornos independentes, ou seja, transtornos que co-ocorrem sem exercerem influência entre si (Rhee, Hewitt, Corley, Willcutt, \& Pennington, 2005).

Independente do tipo de relação existente entre TDAH e TA, diagnósticos de ambos os transtornos são frequentes em crianças que apresentam dificuldades acadêmicas e comportamentais (Hooper \& Williams, 2005). Apesar do avanço das pesquisas nas duas últimas décadas, ainda não há consenso quanto à definição clara para designar o TA, para os quais muitos termos são utilizados. O conceito é amplo e a maioria das crianças é identificada como porta- dora de TA devido ao seu desempenho inesperadamente baixo em um ou mais domínios do desempenho acadêmico, incluindo as áreas de leitura, escrita e matemática (American Psychiatric Association [APA], 2003; Fletcher, Lyons, Fuchs, \& Barnes, 2009).

Refletindo a imprecisão conceitual, encontra-se uma taxa de prevalência muito variável de $15 \%$ a $50 \%$ de TA nas amostras de crianças com TDAH (Riesgo, 2006), taxa essa baseada no DSM-IV-TR. Esse percentual amplo necessita ser refinado a fim de possibilitar uma compreensão mais específica das dificuldades, tendo em vista uma intervenção precisa nos indivíduos com TDAH. Sendo assim, o objetivo desse artigo é descrever e analisar a prevalência do TA, em uma amostra referida de crianças e adolescentes com TDAH diagnosticadas segundo os critérios do DSM-IV-TR (APA, 2003), discutindo tal prevalência no contexto das mudanças nos critérios diagnósticos no DSM-5.

Este artigo é composto por três partes: na primeira parte, revisamos a literatura a respeito da definição do TA e prevalência do TA em crianças e adolescentes com TDAH e apresentamos os critérios de diagnóstico definidos no DSM-5; na segunda parte apresentamos um estudo da distribuição de TA em um grupo de 270 crianças com TDAH de uma amostra referida, de acordo com os critérios do DSM-IV-TR (APA, 2003) e, por último, analisamos os resultados encontrados em tal estudo considerando o contexto das mudanças definidas no DSM-5.

\section{Revisão da Literatura}

Embora o termo "learning disabilities" "venha sendo usado desde 1962, não existe uma única definição universalmente aceita para os transtornos de aprendizagem (Fletcher et al., 2009). A quinta edição do Manual Diagnóstico e Estatístico de Transtornos Mentais (DSM-5), recentemente lançada (APA, 2013), situa os Transtornos de Aprendizagem na secção 2 do Manual, na categoria dos Transtornos do Neurodesenvolvimento_e assim os define:

Transtorno de Aprendizagem Específico é uma desordem neurodesenvolvimental, de origem biológica, que é a base das dificuldades, em nível cognitivo, que estão associadas às expressões comportamentais do transtorno. A origem biológica inclui uma interação de fatores genéticos, epigenéticos e ambientais os quais afetam a habilidade cerebral de perceber ou processar informação verbal ou não-verbal de forma eficiente e precisa. (2013, p. 68)

Esta definição abandona a ideia da discrepância entre quociente de inteligência (QI) e o desempenho acadêmico, presente no DSM-IV-TR (APA, 2003). Os critérios diagnósticos propostos também se diferenciam da versão anterior. Os critérios são classificados em quatro grupos, dos quais apresentamos o primeiro grupo a seguir:

\footnotetext{
${ }^{1}$ Ainda não há consenso quanto à tradução deste termo. Há autores que sugerem Transtorno de Aprendizagem (Moojen, 1996, 2004) e outros que optam por Distúrbios de Aprendizagem (Ciasca, 2003).
} 
Dorneles, B. V., Corso, L. V., Costa, A. C., Pisacco, N. M. T., Sperafico, Y. L. S. \& Rohde, L. A. P. (2014). Impacto do DSM-5 no Diagnóstico de Transtornos de Aprendizagem em Crianças e Adolescentes com TDAH: Um Estudo de Prevalência.

A. Dificuldades na aprendizagem e no uso de habilidades acadêmicas, indicadas pela presença de, pelo menos um dos sintomas seguintes, que persistem por, pelo menos, seis meses, apesar da realização de intervenções dirigidas a essas dificuldades:

1. Imprecisão ou lentidão na leitura de palavras (por exemplo, leitura oral de palavras incorreta ou lenta e hesitante, frequentemente esquece palavras e dificuldades em pronunciar palavras).

2. Dificuldades de compreender o sentido do que é lido (isto é, pode ler um texto corretamente, mas não entender a sequência, as relações, inferências ou sentidos mais profundos do que é lido.)

3. Dificuldades na soletração (por exemplo, pode acrescentar, omitir ou substituir vogais ou consoantes).

4. Dificuldades com expressão escrita (pode cometer erros gramaticais ou de pontuação nas sentenças, utilizar uma organização frágil de parágrafos ou faltar clareza na expressão escrita de ideias).
5. Dificuldades relativas a senso numérico, fatos numéricos ou cálculo (por exemplo, tem pouca compreensão de número, sua magnitude e relações, conta nos dedos para adicionar números pequenos ao invés de recuperar fatos da memória como fazem seus pares, perde-se no processamento aritmético e pode misturar procedimentos).

6. Dificuldades com raciocínio matemático (por exemplo, tem dificuldade severa de aplicar conceitos, fatos ou procedimentos para resolver problemas quantitativos. (2013, p. 66)

As modificações definidas pelo DSM-5 para os critérios diagnósticos do TA são decorrentes de dados obtidos por pesquisas recentes (Cavendish, 2013; DuPaul et al., 2012; Scanlon, 2013; Tannock, 2012). Cabe lembrar que a APA, que elabora e publica o DSM em suas diferentes revisões, formou equipes de acordo com as áreas de estudo para elaboração do DSM-5.

Na Figura 1, resumimos os critérios, presentes no DSM-IV-TR (APA, 2003) e os atuais definidos pelo DSM-5.

\begin{tabular}{ll}
\hline & DSM - IV-TR \\
\hline Nomenclatura & TRANSTORNOS DE \\
& APRENDIZAGEM \\
& Quatro subtipos: \\
Subtipos & - Transtorno da Leitura (TL) \\
& - Transtorno da Matemática (TM) \\
& - Transtorno da Expressão Escrita (TEE) \\
& - Transtorno da Aprendizagem Sem \\
& Outra Especificação
\end{tabular}

Caracterização dos critérios diagnósticos

Específicos para cada subtipo: TL, TM e TEE

Idade inicial para diagnóstico

Baixo desempenho acadêmico

$\mathrm{TL}$ - final da $1^{\mathrm{a}}$ série $/ 2^{\circ}$ ano

$\mathrm{TM}$ - final da $2^{\mathrm{a}}$ série $/ 3^{\circ}$ ano

TEE $-2^{\mathrm{a}}$ série $/ 3^{\mathrm{o}}$ ano

Desempenho substancialmente abaixo do esperado considerando a idade,
DSM- 5- Alterações:

\section{TRANSTORNO DE APRENDIZAGEM}

Categoria única:

- Transtorno de Aprendizagem

(preserva as distinções e continuidades entre as várias manifestações dos transtornos específicos de aprendizagem)

Gerais para TA e uso de especificadores do desenvolvimento dos três principais domínios acadêmicos (leitura, escrita e matemática).

Primeiros anos de escolaridade, ou, nos anos seguintes, quando as demandas da aprendizagem excedam as limitadas capacidades individuais. escolarização e nível de inteligência do indivíduo.
Desempenho substancialmente abaixo, em uma ou mais áreas acadêmicas, considerando a média esperada para a idade (também são referidos como padrões a serem levados em consideração o contexto cultural, linguístico, ou sexo, ou nível de educação (substituição de $e$ por $o u$ )

- Acréscimo de quesitos: medidas padronizadas e quantificação de desempenho acadêmico com aplicação de testes individuais.

- Maior consideração às evidências clínicas 
Persistência

Resposta à intervenção - RTI

Discrepância rendimento/QI

Exclusão/Inclusão
Descrição genérica da persistência.

No TL, com a intervenção precoce o prognóstico é bom em uma percentagem significativa de casos. Mas pode persistir até a vida adulta.

Não especifica no TM.

TEE pode ser visto em crianças mais velhas e adultos, mas pouco se sabe sobre seu prognóstico.

A persistência de dificuldades da aprendizagem é citada.

Desmoralização, baixa autoestima e déficits nas habilidades sociais podem ser expressão dos prejuízos que o TA causa no desenvolvimento.

Rendimento substancialmente abaixo do QI

As dificuldades de aprendizagem não são melhor explicadas por déficit intelectual, condição neurológica, outra condição médica geral ou outro déficit sensorial.
A persistência é incluída como critério diagnóstico: história ou apresentação de dificuldades persistentes, por um período mínimo de seis meses, na aquisição de leitura, escrita, aritmética, ou habilidades de raciocínio matemático durante os anos de estudo formais (ou seja, durante o período de desenvolvimento).

Dificuldades de aprendizagem identificadas no Critério A:

- persistem apesar da intervenção focada nos problemas manifestados por um ou mais dos sintomas clínicos/ comportamentais;

- não podem ser explicadas pela ausência das ferramentas, suportes, ou serviços fornecidos para a compensação dessas dificuldades.

Elimina o critério de discrepância como geral, mantendo o QI como critério de exclusão.

As dificuldades de aprendizagem não são melhor explicadas por Transtorno do Desenvolvimento Intelectual, atraso no desenvolvimento global, outros transtornos de ordem neurológica, sensorial (visão, audição), distúrbios motores, adversidade psicossocial, ou falta de oportunidade de ensino.

Figura 1. Quadro comparativo dos critérios diagnósticos do TA no DSM -IV - TR e no DSM -5 (APA, 2003, 2010, 2013).

A discussão dessas alterações é muito importante, pois, como alertam Cavendish (2013) e Tannock (2012), os critérios diagnósticos do DSM-IV-TR para os TA deram origem a preocupações clínicas, já que a utilização da discrepância entre QI e desempenho como critério único, sem o uso de outras medidas padronizadas, foi bastante questionada, pois o baixo desempenho pode estar relacionado à inadequação pedagógica. Além disso, há uma base de pesquisa crescente mostrando a eficácia das intervenções precoces nessas crianças com baixo desempenho. Neste contexto, tem-se discutido a importância e a viabilidade do uso da Resposta à Intervenção (RTI), que tem sido defendida como mais um critério diagnóstico de alunos com dificuldades persistentes na aprendizagem por alguns pesquisadores (Almeida, Pizza, \& Miranda, 2011; Fuchs, Fuchs, \& Hollenbeck, 2007; Gersten et al., 2009).

A RTI é definida por Gersten e colaboradores (2009) como um sistema de apoio, de detecção precoce e intervenção que tem como objetivo identificar alunos que apresentam baixo desempenho, assistindo-os para que progridam em sua aprendizagem. Essa abordagem tem como hipótese central a ideia de que um estudante que não demonstra progresso após completar diversas sessões de intervenção é um provável portador de transtorno de aprendizagem. Já os estudantes que avançam com o passar das sessões, são identificados como não sendo portadores de transtorno de aprendizagem e seu baixo desempenho pode ser resultado de uma instrução pobre em sala de aula (Fuchs et al., 2007). Dessa forma, tal modalidade de intervenção é eficaz para diferenciar estudantes com possíveis transtornos de aprendizagem daqueles que possuem baixo desempenho devido à instrução inadequada recebida em sala de aula. Entretanto, ainda não há consenso quanto a RTI como um bom critério diagnóstico de TA. Além disso, essa abordagem introduz um parâmetro novo no DSM, ou seja, esse passa a ser o único transtorno presente no Manual para o qual é utilizado um dado de tratamento para definir o diagnóstico.

As mudanças nos critérios do DSM-5 para a identificação do TA, em especial o RTI, trarão implicações para a atuação, durante a avaliação diagnóstica, não apenas para os médicos, mas também aos psicólogos, psicopedagogos e professores (Scanlon, 2013). A principal mudança que o DSM-5 apresenta é uma mudança de visão quanto ao diagnóstico de TA: a ênfase deixa de ser a discrepância da habilidade de leitura, escrita ou matemática em relação ao QI e passa para o desempenho inadequado da habilidade de acordo com testes padronizados para sexo, idade, escolaridade ou grupos culturais ou linguísticos. O maior problema que tal mudança traz para países como o Brasil 
Dorneles, B. V., Corso, L. V., Costa, A. C., Pisacco, N. M. T., Sperafico, Y. L. S. \& Rohde, L. A. P. (2014). Impacto do DSM-5 no Diagnóstico de Transtornos de Aprendizagem em Crianças e Adolescentes com TDAH: Um Estudo de Prevalência.

diz respeito à ausência total de testes padronizados quanto à idade, nível de escolaridade, grupo cultural ou grupo linguístico, que sirvam para as diferentes regiões do país, o que praticamente inviabiliza um diagnóstico de TA.

Posto isso, apresentamos um estudo da distribuição de TA em um grupo de 270 crianças com TDAH de uma amostra referida, avaliados com base nos critérios diagnósticos do DSM-IV-TR, buscando, na sequência, discutir o impacto que o DSM-5 terá na prevalência de tais transtornos.

\section{Método}

\section{Descrição da Amostra}

A amostra do estudo foi composta por 270 crianças e adolescentes de 8 a 17 anos de idade que participaram ou continuam participando do Programa de Transtornos de Déficit de Atenção/Hiperatividade (ProDAH). O ProDAH é um programa que faz parte do Serviço de Psiquiatria da Infância e Adolescência e do Serviço de Psiquiatria do Hospital de Clínicas de Porto Alegre (HCPA) e do Departamento de Psiquiatria e Medicina Legal da Universidade Federal do Rio Grande do Sul (UFRGS), composta por uma equipe multidisciplinar dedicada ao ensino, pesquisa, extensão e atendimento a pacientes com o transtorno. Os sujeitos foram selecionados no banco de dados do ProDAH tendo como critérios de inclusão: (a) possuírem diagnóstico de TDAH de qualquer tipo, confirmado pela equipe do ProDAH, de acordo com os critérios do DSM-IV-TR (APA, 2003); (b) terem QI estimado (WISC-III; Wechsler, 2002) acima de 80; (c) estarem cursando do terceiro ano do Ensino Fundamental ao terceiro ano do Ensino Médio; e (d) possuírem avaliação psicopedagógica, realizada pela equipe de psicopedagogos do ProDAH, para verificação da existência de TA.

\section{Procedimentos de Avaliação}

Processo Diagnóstico do TDAH. O diagnóstico do TDAH e de suas comorbidades foi realizado na unidade ambulatorial do ProDAH, seguindo os procedimentos já descritos (Rohde, 2002) e utilizados em outras pesquisas (Polanczyk, 2005). O processo ocorre em três fases: (a) avaliação com uma entrevista semiestruturada (Schedule for Affective Disorders and Schizophrenia for School-Age Children- Epidemiologic Version [K-SADS-E $\left.{ }^{2}\right]$ ); (b) discussão diagnóstica em um comitê clínico, coordenado por um dos médicos do ProDAH e (c) avaliação clínica.

Avaliação do Nivel Intelectual. A avaliação foi realizada na unidade ambulatorial do ProDAH, por psicólogas treinadas, através dos subtestes vocabulário e cubos (WISC III; Wechsler, 2002). A análise combinada de ambos possi-

\footnotetext{
${ }^{2} \mathrm{O}$ K-SADS-E verifica e registra episódios psicopatológicos, passados ou atuais, em crianças e adolescentes, segundo critérios do DSM-III-R e DSM-IV, traduzido para o português por Mercadante e colaboradores (1995 apud Mercadante, 1999).
}

bilitou a obtenção de um QI ponderado das crianças e adolescentes analisados, considerando a escala de pontuação do teste de acordo com as idades das crianças. A escolha desses dois subtestes, um verbal e outro de execução, para a avaliação do QI estimado já vem sendo utilizada em outras pesquisas (Geary \& Hoard, 2001; Geary, Hoard, \& Hamson, 1999).

Identificação dos Transtornos de Aprendizagem. Como já referimos, no Brasil, ainda há indefinição quanto à natureza, validade e fidedignidade dos instrumentos de avaliação dos transtornos de aprendizagem. Tal assunto foi amplamente discutido, por exemplo, em Corso (2008) e Salles (2004). A escolha dos instrumentos seguiu aqueles comumente usados na avaliação psicopedagógica (Moojen \& Costa, 2006). Todos os instrumentos foram aplicados pela equipe de psicopedagogia. Mesmo o diagnóstico sendo multidisciplinar, enfatizamos os instrumentos psicopedagógicos, pois eles foram essenciais para o diagnóstico dos Transtornos de Aprendizagem.

O modelo de classificação dos transtornos de aprendizagem foi o da discrepância entre QI e desempenho escolar proposto pelo DSM-IV-TR (APA, 2003). A avaliação psicopedagógica fornece os indicadores que vão mostrar a discrepância entre o potencial intelectual individual e o desempenho acadêmico em relação ao que seria esperado para a escolaridade. O ponto de corte adotado foi de dois anos de atraso com relação ao esperado para a faixa de escolaridade, em cada um dos domínios avaliados.

$\mathrm{Na}$ aritmética, o instrumento utilizado foi o Sub-teste de Aritmética, organizado e padronizado por Stein (1994), composto de cálculos aritméticos com grau de dificuldade crescente, correspondentes ao conteúdo de $1^{\mathrm{a}}$ a $6^{\mathrm{a}}$ série. Os resultados foram analisados seguindo a padronização do próprio teste.

$\mathrm{Na}$ escrita foi avaliada somente a ortografia, através de um ditado de 50 palavras, proposto por Moojen (2009), e avaliado conforme a padronização proposta pela autora.

$\mathrm{Na}$ leitura, foram avaliadas três habilidades: via fonológica, fluência e compreensão leitora. As vias de reconhecimento foram avaliadas através de dois protocolos: de decodificação de sílabas complexas (Moojen, 2001) e de decodificação de palavras e pseudopalavras (Moojen \& Costa, 2003). Foram realizadas duas análises: quantitativa (um ponto para cada palavra ou sílaba lida de forma correta) e qualitativa (tipo de erro).

A fluência e a compreensão leitora foram avaliadas pelo instrumento proposto por Saraiva, Moojen e Munarski (2007) e analisado conforme indicação das autoras.

\section{Resultados e Discussão}

O objetivo inicial da pesquisa foi definir a prevalência de TA em uma amostra referida de 270 crianças e adolescentes com TDAH. Sendo assim, realizou-se, primeiramente, um levantamento quanto ao tipo de TDAH apresentado pela amostra, como mostra a Figura 2. 


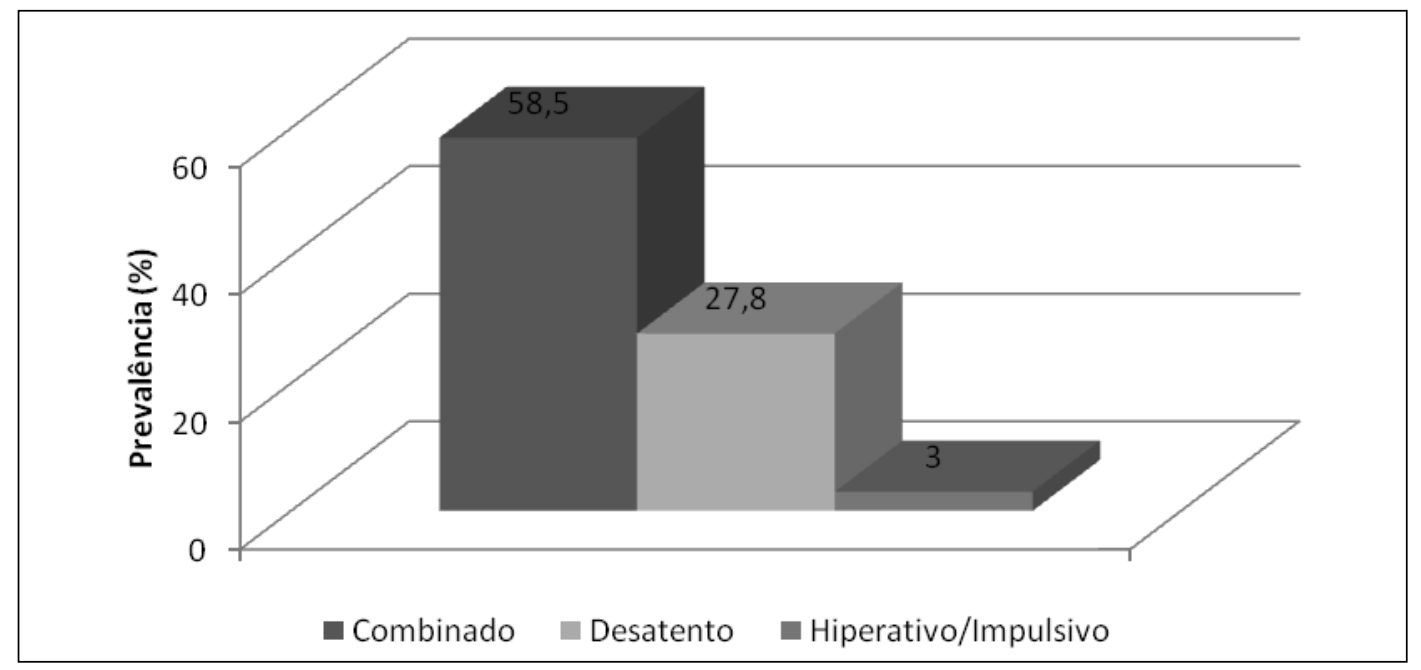

Figura 2. Prevalência do tipo de TDAH em amostra de 270 crianças.

O tipo predominante de TDAH na amostra investigada é o combinado (58,5\%), seguido do tipo desatento $(27,8 \%)$, e as taxas de prevalência são semelhantes às apresentadas na revisão de Zentall (2007). O TDAH tipo hiperativo/ impulsivo aparece como o menos frequente, mas apresenta um percentual de prevalência bastante inferior ao relatado no estudo de Zentall (2007), que foi de $15 \%$. Esse fato pode ser justificado pela amostra de crianças e adolescentes selecionados, que buscam um atendimento especializado quando apresentam sintomas que provocam prejuízos, muitos deles escolares. Sendo assim, parece que os tipos desatento e combinado são os que mais impacto tem na realização acadêmica, devido ao sintoma de desatenção (DuPaul et al., 2012; Zentall, 2007), o que pode ser a causa de um maior número de atendimentos de indivíduos por- tadores de TDAH-Desatento ou TDAH-Combinado. Ao mesmo tempo, sintomas de hiperatividade-impulsividade parecem acarretar maior incidência de problemas relacionados a aspectos comportamentais, afetando as interações sociais na escola (Wu \& Gau, 2013).

Verificou-se também a prevalência de TA na amostra, encontrando-se um percentual de $46,7 \%$, que está localizado na extremidade superior do intervalo de percentuais sugerido por Riesgo (2006) de $15 \%$ a 50\%. Salienta-se que, dos 126 sujeitos acometidos pela comorbidade, 53,17\% apresentam apenas um tipo de TA, 42,86\% apresentam dois transtornos e 3,97\% apresentam os três tipos de TA. A seguir especificou-se a prevalência quanto ao tipo de TA apresentado pelos estudantes, em Transtorno de Leitura (TL), Transtorno da Expressão Escrita (TEE) e Transtorno da Matemática (TM), resumida na Figura 3.

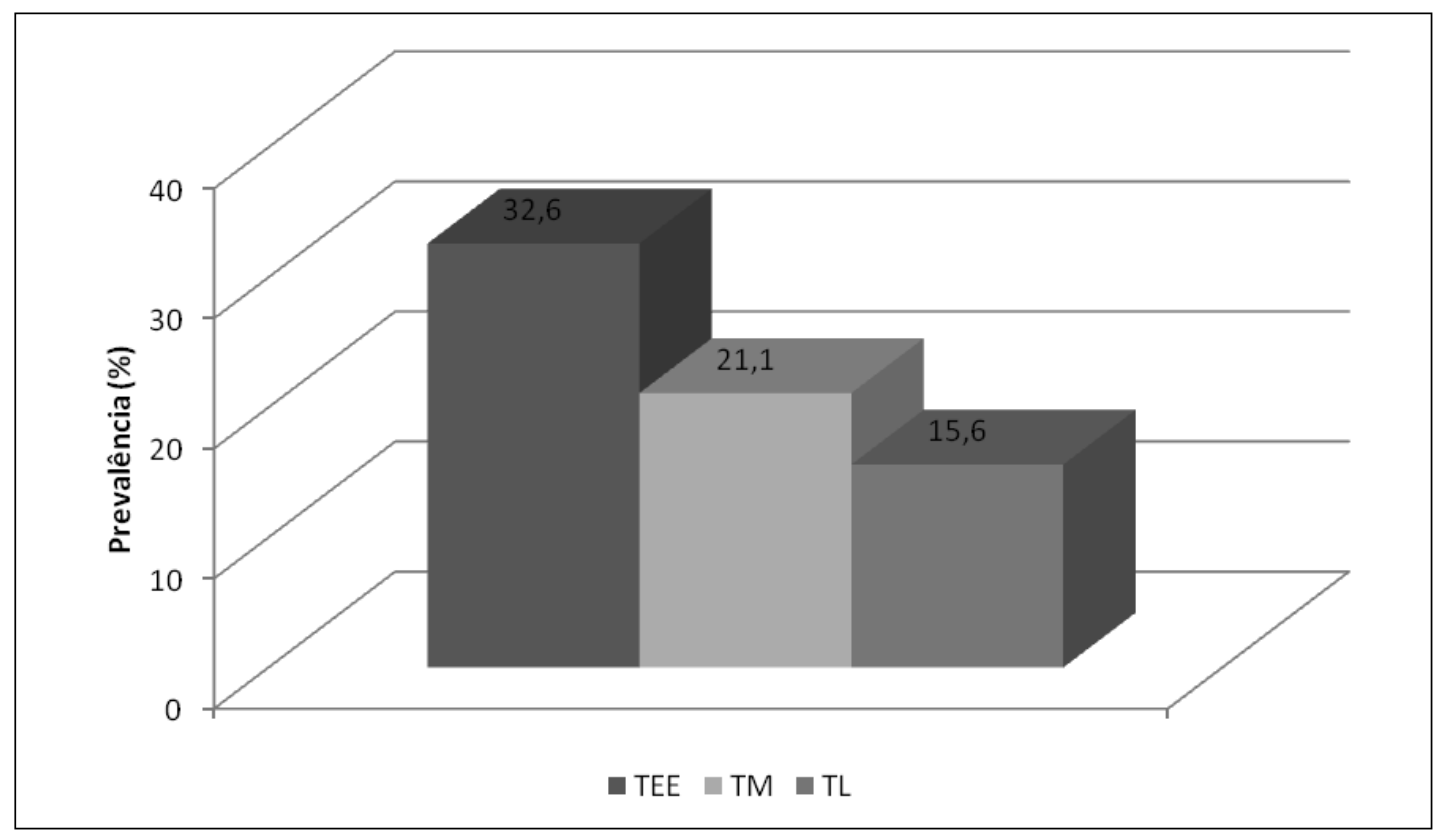

Figura 2. Porcentagem de TA no TDAH. 
Dorneles, B. V., Corso, L. V., Costa, A. C., Pisacco, N. M. T., Sperafico, Y. L. S. \& Rohde, L. A. P. (2014). Impacto do DSM-5 no Diagnóstico de Transtornos de Aprendizagem em Crianças e Adolescentes com TDAH: Um Estudo de Prevalência.

O TEE, mais frequente no TDAH (DuPaul et al., 2012; Mayes \& Calhoun, 2006; Rohde et al., 2006), também é o que apresenta a maior taxa de prevalência, $32,6 \%$, na amostra analisada. O TM aparece como o segundo mais frequente, $21,1 \%$, apresentando uma taxa de prevalência dentro do intervalo já encontrado na literatura, de $5 \%$ a

Tabela 1

Prevalência de TA nos Tipos de TDAH

\begin{tabular}{lcccccc}
\hline \multirow{2}{*}{ Subtipo de TDAH } & \multicolumn{2}{c}{ TL } & \multicolumn{2}{c}{ TEE } & \multicolumn{2}{c}{ TM } \\
\cline { 2 - 7 } & $N$ & $\%$ & $N$ & $\%$ & $N$ & $\%$ \\
\hline Desatento & 17 & 40,5 & 27 & 30,7 & 18 & 31,6 \\
Hiperativo/impulsivo & 0 & 0 & 0 & 0 & 1 & 1,8 \\
Combinado & 21 & 50 & 52 & 59,1 & 28 & 49,1 \\
Total & 42 & 100 & 88 & 100 & 57 & 100 \\
\hline
\end{tabular}

Nota. Fonte: banco de dados do ProDAH (2009).

Observa-se, na Tabela 1, que o tipo combinado, seguido do tipo desatento, são os que apresentam as maiores taxas de TA. Esse dado vai ao encontro dos estudos revisados que indicam ser o sintoma de desatenção o que mais tem impacto sobre a realização acadêmica dos estudantes (DuPaul et al., 2012; Lee et al., 2008; Marshall, Schafer, O’Donnell, \& Handwerk, 1999; Wu \& Gau, 2013; Zentall, 2007).

Os dados acima apresentados ajudam a refinar os percentuais de prevalência do TA no TDAH, especificando as taxas para cada tipo de TA. Entretanto, as mudanças no diagnóstico do TA, propostas para o DSM-5, podem trazer alterações nessas taxas, como discutiremos a seguir.

Discussão dos Resultados no Contexto das Propostas de Mudança nos Critérios Diagnósticos para o DSM-5

$\mathrm{O}$ resultado que cada uma das mudanças nos critérios diagnósticos de TA trará sobre a prevalência de comorbidade entre TDAH e TA ainda é incerto. Entretanto, é possível levantar algumas hipóteses.

Quanto ao desempenho acadêmico, a substituição da conjunção $e$ pela conjunção $o u$ poderá elevar a taxa de prevalência do diagnóstico. No DSM-IV-TR, para uma criança ser diagnosticada com TA, seu desempenho deveria estar abaixo do esperado considerando a idade, escolarização $e$ nível de inteligência do indivíduo. Assim, na mudança, o desempenho do aluno pode ser incompatível com um dos critérios e ainda assim ser possível fechar o diagnóstico de TA (DuPaul et al., 2012). O aspecto mais significativo nos parece ser o abandono do papel fundamental da discrepância entre QI e habilidade que foi substituída pela ideia de noção de desempenho abaixo do esperado em testes padronizados para idade, cultura e escolaridade. Dito de outra forma, quando ambos (QI e habilidade) estão baixos, mas QI ainda não é compatível com deficiência intelectual, pelo DSM-5 se confirma o diagnóstico de TA, o que não era feito pelos critérios do DSM-IV-R. Considerando-se a escassez de testes padronizados de habilidades de escrita, matemática e leitura,
$30 \%$. O TL também está em consonância com o intervalo descrito na literatura, $11 \%$ a $52 \%$ (DuPaul et al., 2012; Mayes \& Calhoun, 2006), sendo encontrado em 15,6\% dos estudantes com TDAH da amostra.

Buscou-se ainda realizar uma descrição das taxas de prevalência dos TA em cada tipo de TDAH, resumida na Tabela 1. de acordo com a escolaridade, na realidade brasileira, tal critério torna-se difícil de ser aplicado da mesma forma em todas as regiões do país, com diferentes culturas e maneiras de ensinar.

Por outro lado, a exigência de múltiplas medidas de avaliação, bem como a indicação de verificação da existência de dificuldades de aprendizagem por um período mínimo de seis meses, persistentes mesmo após intervenções focadas no problema de aprendizagem, podem diminuir a prevalência. Concordamos com Tannock (2012) quanto ao fato de que esses critérios permitem tornar o diagnóstico de TA mais criterioso e confiável. Entretanto, esta alteração impedirá um diagnóstico rápido, uma vez que a presença do TA só poderá ser estabelecida após seis meses de tratamento específico.

Cabe relembrar que a validade do RTI como método de identificação de transtornos não é consensual, já que há variabilidade nas medidas de avaliação, utilizadas no processo de triagem das dificuldades, e, especialmente, nas propostas de intervenção. Questiona-se também a falta de base empírica para determinar as medidas baseadas no currículo ou sua confiabilidade e validade para a realização desse diagnóstico inicial (Cavendish, 2013). Assim, apesar dos benefícios da RTI apontados na literatura, ainda há muitas dúvidas quanto ao seu uso como método de identificação de TA, o que talvez justifique o fato de que esta é uma forma de diagnóstico não utilizada em nenhuma outra área do DSM-5. Assim, tal critério torna o diagnóstico inviável em países como o Brasil onde só uma parcela da população tem acesso à avaliação e intervenção. A implementação da RTI como critério pressupõe uma eficiente formação de profissionais, terapeutas e professores que possibilite uma intervenção individual e adequada orientação psicopedagógica às escolas, dirigida às dificuldades específicas de cada criança, formação essa que ainda não é comum no Brasil.

Posto isso, pode-se afirmar que as mudanças discutidas podem trazer alteração nas taxas apresentadas no estudo 
acima. No entanto, a combinação dos principais critérios (ou ao invés de $e$, e resposta à intervenção) parece restringir a possibilidade de um aumento significativo nas taxas de prevalência. Parece-nos, então, que pode haver uma permanência das atuais taxas de comorbidade entre TDAH e TA, permanência esta que deverá ser confirmada por estudos futuros.

Cabe destacar, tal como DuPaul e colaboradores (2012) alertam, que, independente das alterações propostas para os critérios diagnósticos no DSM-5, a maioria das crianças com TDAH não terá TA e vice-versa. No entanto, o fato de um grupo de $46,7 \%$ ter a comorbidade em uma amostra clínica deve ser considerado no planejamento escolar e nos programas de intervenção educacionais, uma vez que há evidências (Barkley, 2008) de que, quando os dois transtornos ocorrem juntos, os estudantes demonstraram tanto déficits atencionais quanto acadêmicos mais acentuados do que os daquelas crianças que tem somente um dos transtornos.

Salientamos que a amostra do ProDAH não é representativa da população, em geral, na medida em que é uma amostra referida e mais, considerando que o ProDAH faz parte de um hospital terciário, somente os casos mais graves chegam para atendimento, inflando as taxas de comorbidade.

\section{Considerações Finais}

O presente artigo teve como objetivo definir os percentuais de prevalência de comorbidade entre TDAH e TA, apresentando os resultados de um estudo realizado com uma amostra referida de 270 crianças com TDAH, diagnosticadas conforme os critérios do DSM-IV-TR, bem como discutir o impacto das mudanças propostas no DSM-5 em tais taxas de prevalência. Verificamos uma alta taxa de comorbidade $(46,7 \%)$ entre os dois transtornos, sendo o Transtorno da Expressão Escrita (TEE) o mais prevalente $(32,6 \%)$, resultado que segue a tendência de estudos anteriores.

A análise desses resultados no contexto das mudanças propostas para o DSM-5 permite afirmar que poderão ocorrer alterações nas taxas de prevalência de comorbidade entre TDAH e TA. Entretanto, o efeito que essas mudanças terão sobre a prevalência, especificidade e comorbidade dos transtornos de aprendizagem permanece incerto, na medida em que alguns dos critérios poderão ter um efeito ampliador e outros, ao contrário, tendem a restringir os sintomas característicos de cada TA.

\section{Referências}

Almeida, R., Pizza, C., \& Miranda, M. (2011). Resposta à intervenção: Uma abordagem para prevenir e remediar dificuldades de aprendizagem. Revista Crescimento e Desenvolvimento Humano, 21(2).

American Psychiatric Association. (2003). DSM-4-TR: Manual diagnóstico e estatístico de transtornos mentais (ed. rev.). Porto Alegre, RS: Artes Médicas.
American Psychiatric Association. (2010). DSM-5: Options being considered for $A D H D$. Retrieved from http://www. dsm5.org/ProposedRevisions/Pages/InfancyChildhoodAdolescence.aspx

American Psychiatric Association. (2013). Highlights of changes from DSM-IV-TR to DSM-5. Retrieved from http://www.psychiatry.org/File\%20Library/Practice/DSM/DSM-5/Changesfrom-DSM-IV-TR--to-DSM-5

Barkley, R. (2008). Transtorno de déficit de atenção/hiperatividade (3. ed.). Porto Alegre, RS: Artmed.

Carroll, J. M., Maughan, B., Goodman, R., \& Meltzer, H. (2005). Literacy difficulties and psychiatric disorders: Evidence for comorbidity. Journal of Child Psychology and Psychiatry, 46(5), 524-532. doi:10.1111/j.1469-7610.2004.00366.x

Cavendish, W. (2013). Identification of learning disabilities: Implications of proposed DSM-5 criteria for school-based assessment. Journal of Learning Disabilities, 46(1), 52-57. doi:10.1177/0022219412464352

Ciasca, S. M. (2003). Distúrbios de aprendizagem: Propostas de avaliação interdisciplinar. São Paulo, SP: Casa do Psicólogo.

Corso, L. (2008). Dificuldades na leitura e na escrita: Um estudo dos processos cognitivos em alunos da $3^{a} a 6^{a}$ série do ensino fundamental (Tese de doutorado, Faculdade de Educação, Universidade Federal do Rio Grande do Sul, Porto Alegre, RS, Brasil).

Costa, A. C. (2009). Ensino de fatos básicos aditivos para crianças com Transtorno de Déficit de Atenção/Hiperatividade (TDAH): Possibilidades de intervenção pedagógica na aritmética (Tese de doutorado, Faculdade de Educação, Universidade Federal do Rio Grande do Sul, Porto Alegre, RS, Brasil).

DuPaul, G., Gormley, M., \& Laracy, L. (2012). Comorbidity of LD and ADHD: Implications of DSM-5 for assessment and treatment. Journal of Learning Disabilities, 1-9. doi:10.1177/0022219412464351.

DuPaul, G. J., \& Stoner, G. (2007). TDAH nas escolas. São Paulo, SP: M. Books.

Faraone, S. V., Biederman, J., Monuteaux, M. C., Doyle, A. E., \& Seidman, L. J. (2001). A psychometric measure of learning disability predicts educational failure four years later in boys with attention-deficit/hyperactivity disorder. Journal of Attention Disorders, 4(4), 220-230. doi:10.1177/108705470100400404

Fletcher, J. M., Lyons, G. R., Fuchs, L. S., \& Barnes, M. A. (2009). Transtornos de aprendizagem: Da identificação à prevenção. Porto Alegre, RS: Artmed.

Fuchs, L., Fuchs, D., \& Hollenbeck, K. (2007). Extending responsiveness to intervention to mathematics at first and third grades. Learning Disabilities Research \& Practice, 13-24. doi:10.1111/j.1540-5826.2007.00227.x

Geary, D. C., \& Hoard, M. K. (2001). Numerical and arithmetical deficits in learning-disabled children: Relation to dyscalculia and dyslexia. Aphasiology, 15(7), 635-647. doi: $10.1080 / 02687040143000113$

Geary, D. C., Hoard, M. K., \& Hamson, C. O. (1999). Numerical and arithmetical cognition: Patterns of functions and deficits in children at risk for mathematical disability. Journal of Experimental Child Psychology, 74, 213-239. doi:10.1006/ jecp. 1999.2515

Gersten, R., Beckmann, S., Clarke, B., Foegen, A., Marsh, L., Star, J. R., \& Witzel, B. (2009). Assisting students struggling with mathematics: Response to intervention (RtI) for elementary and middle schools. Washington, DC: National Center for Education Evaluation and Regional Assistance, U.S. Department of Education. Retrieved from http://www.opi. mt.gov/pub/RTI/Training/IESMathPracticeGuide.pdf 
Dorneles, B. V., Corso, L. V., Costa, A. C., Pisacco, N. M. T., Sperafico, Y. L. S. \& Rohde, L. A. P. (2014). Impacto do DSM-5 no Diagnóstico de Transtornos de Aprendizagem em Crianças e Adolescentes com TDAH: Um Estudo de Prevalência.

Hooper, S. R., \& Williams, E. A. (2005). Attention deficit hyperactivity disorder and learning disabilities. In D. Gozal \& D. L. Molfese, Attention deficit hyperactivity disorder: From genes to patients (pp. 215-254). Totowa, NJ: Humana Press.

Lee, S. S., Lahey, B. B., Owens, E. B., \& Hinshaw, S. P. (2008). Few preschool boys and girls with ADHD dare well-adjusted during adolescence. Journal of Abnormal Child Psychology, 36, 373-383. doi:10.1007/s10802-007-9184-6

Marshall, R. M., Schafer, V. A., O’Donnell, J. E., \& Handwerk, M. L. (1999). Arithmetic disabilities and add subtypes: Implications for DSM-IV. Journal of Learning Disabilities, 32(3), 239-247. doi:10.1177/002221949903200305

Martinussen, R., Hayden, J., Hogg-Johnson, S., \& Tannock, R. (2005). A meta-analysis of working memory impairments in children with attention-deficit/hyperactivity disorder. Journal of the American Academy of Child and Adolescent Psychiatry, 44(4), 377-384. doi:10.1097/01.chi.0000153228.72591.73

Mayes, S., \& Calhoun, S. (2006). Frequency of reading, math, and writing disabilities in children with clinical disorders. Learning and Individual Differences, 16(2), 145-157. doi:10.1016/j.lindif.2005.07.004

Mayes, S., Calhoun, S., \& Crowell, E. (2000). Learning disabilities and ADHD: Overlapping spectrum disorders. Journal of Learning Disabilities, 33(5), 417-424. doi:10.1177/002221940003300502

Mercadante, M. T. (1999). Transtorno obsessivo-compulsivo, tiques, Síndrome de Tourette e outros transtornos psiquiátricos em pacientes com febre reumática, com ou sem Coréia de Sydenham (Tese de doutorado, Programa de Pós-Graduação em Psiquiatria, Faculdade de Medicina, Universidade de São Paulo, SP, Brasil).

Moojen, S. (1996). Dificuldades na aprendizagem escolar. In P. B. Sukiennik (Ed.), O aluno problema: Transtornos emocionais de crianças e adolescentes (pp. 79-110). Porto Alegre, RS: Mercado Aberto.

Moojen, S. (2001). Protocolo de decodificação de sílabas complexas. Trabalho não publicado.

Moojen, S. (2004). Diagnósticos em Psicopedagogia. Revista Psicopedagogia (São Paulo), 66, 245-255.

Moojen, S. (2009). A escrita ortográfica na escola e na clínica: Teoria, avaliação e tratamento. São Paulo, SP: Casa do Psicólogo.

Moojen, S., \& Costa, A. (2003). Protocolo de decodificação de palavras e pseudopalavras. Trabalho não publicado.

Moojen, S., \& Costa, A. (2006). Semiologia psicopedagógica. In N. Rotta, L. Ohlweiler, \& R. S. Riesgo (Eds.), Transtornos da aprendizagem: Abordagem neurobiológica e multidisciplinar (pp. 103-112). Porto Alegre, RS: Artmed.

Polanczyk, G. V. (2005). A associação entre genes do sistema noradrenégico e a resposta clínica ao tratamento com metilfenidato em crianças e adolescentes com transtorno de déficit de atenção/hiperatividade: Um estudo de farmacogenética (Dissertação de mestrado, Faculdade de Medicina, Universidade Federal do Rio Grande do Sul, Porto Alegre, RS, Brasil).

Polanczyk, G., de Lima M. S., Horta, B. L., Biederman, J., \& Rohde, L. A. (2007). The worldwide prevalence of ADHD: A systematic review and metaregression analysis. American Journal of Psychiatry, 164(6), 942-948. doi:10.1176/appi. ajp.164.6.942

Programa de Transtornos de Déficit de Atenção/Hiperatitividade. (2009). ProDAH: Programa de transtornos de déficit de atenção/hiperatitividade. Porto Alegre, RS: Hospital de Clínicas de Porto Alegre, Universidade Federal do Rio Grande do Sul. Recuperado em http://www.ufrgs.br/prodah/

Rhee, S. H., Hewitt, J. K., Corley, R. P., Willcutt, E. G., \& Pennington, B. F. (2005). Testing hypotheses regarding the causes of comorbidity: Examining the underlying deficits of comorbid disorders. Journal of Abnormal Psychology, 114(3), 346-362. doi:10.1037/0021-843X.114.3.346

Rief, S. (2006). The ADD/ADHD checklist: A practical reference for parents and teachers. San Francisco, CA: Jossey Bass.

Riesgo, R. S. (2006). Transtornos de atenção: Co-morbidades. In N. T. Rotta, L. Ohlweilwe, \& R. S. Riesgo (Eds.), Transtornos da aprendizagem: Abordagem neurobiológica e multidisciplinar (pp. 347-363). Porto Alegre, RS: Artmed.

Rohde, L. A. (2002). ADHD in Brazil: The DSM-IV criteria in a culturally different population. Journal of the American Academy of Child and Adolescent Psychiatry, 41(9), 11311133. doi:10.1097/00004583-200209000-00014

Rohde, L. A, Biederman, J., Busnello, E. A., Zimmermann, H., Schmitz, M., Martins, S., \& Tramontina, S. (1999). ADHD in a school sample of Brazilian adolescents: A study of prevalence, comorbid conditions, and impairments. Journal of the American Academy of Child and Adolescent Psychiatry, 38, 716-722. doi:10.1097/00004583-199906000-00019

Rohde, L. A., Dorneles, B. V., \& Costa, A. C. (2006). Intervenções escolares no transtorno de déficit de atenção/hiperatividade. In N. T. Rotta, L. Ohlweilwe, \& R. S. Riesgo (Eds.), Transtornos da aprendizagem: Abordagem neurobiológica e multidisciplinar (pp. 365-374). Porto Alegre, RS: Artmed.

Salles, J. F. (2004). Habilidades e dificuldades de leitura e escrita em crianças de $2^{a}$ série: Abordagem neuropsicológica cognitiva (Tese de doutorado, Instituto de Psicologia, Universidade Federal do Rio Grande do Sul, Porto Alegre, RS, Brasil).

Saraiva, R., Moojen, S., \& Munarski, R. (2007). Avaliação da compreensão leitora: Textos expositivos. Porto Alegre, RS: Casa do Psicólogo.

Scanlon, D. (2013). Specific learning disability and its newest definition: Which is comprehensive? And which is insufficient? Journal of Learning Disabilities, 46(1), 26-33. doi:10.1177/0022219412464342

Stein, L. (1994). TDE - Teste de Desempenho Escolar: Manual para a aplicação e interpretação. São Paulo, SP: Casa do Psicólogo.

Tannock, R. (2012). Rethinking ADHD and LD in DSM-5: Proposed changes in diagnostic criteria. Journal of Learning Disabilities, 1-21. doi:10.1177/0022219412464341

Wechsler, D. (2002). WISC-III: Escala de Inteligência Wechsler para Crianças: Manual (3. ed., V. L. M. de Figueiredo, Adaptação e Padronização Brasileira). São Paulo, SP: Casa do Psicólogo.

Willcutt, E. G., Pennington, B. F., Olson, R. K., Chhabildas, N., \& Hulslander, J. (2005). Neuropsychological analyses of comorbidity between reading disability and attention deficit hyperactivity disorder: In search of the common deficit. Developmental Neuropsychology, 27(1), 35-78. doi:10.1207/ s15326942dn2701 3

Wu, S., \& Gau, S. S. (2013). Correlates for academic performance and school functioning among youths with and without persistent attention-deficit/hyperactivity disorder. Research in Developmental Disabilities, 34, 505-515. doi:10.1016/j. ridd.2012.09.004

Zentall, S. S. (2007). Math performance of students with ADHD: Cognitive and behavioral contributors and interventions. In D. B. Berch \& M. M. M. Mazzocco, Why is math so hard for some children? (pp. 219-243). Baltimore, MD: Brookes. 